\title{
El estudio de los procesos con verbos de movimiento en el quechua chanka
}

\section{The Study of Processes with Verbs of Movement in Chanka Quechua}

\author{
Mery Eli Ascencio Huaccho \\ Universidad Nacional Mayor de San Marcos, \\ Lima, Perú \\ mery.ascencio@unmsm.edu.pe \\ oRCID https://orcid.org/oooo-0oo2-8217-6135
}

\author{
Raquel Herlinda Román Barrera \\ Universidad Nacional Mayor de San Marcos, \\ Lima, Perú \\ raquel.roman@unmsm.edu.pe \\ oRCID https://orcid.org/oooo-0oo2-4241-9438
}

\section{Resumen}

En este trabajo se exponen, brevemente, los procesos expresados con verbos de movimiento en el quechua Chanka, de la zona de Ayacucho. Debido a que el quechua es una lengua aglutinante, este trabajo tiene por objetivo analizar la función de los sufijos desde el ámbito semántico. Para ello, nuestro marco teórico se basa en el aporte de la semántica cognitiva y en la relación entre semántica y gramática propuesta por Moreno Cabrera (2003). Los resultados de este trabajo pretenden clasificar y determinar cómo actúan los sufijos -man, -manta, -kama y -ta en construcciones oracionales de movimiento.

Palabras clave: semántica cognitiva, semántica formal, proceso, verbos de movimiento, verbos de desplazamiento.

\begin{abstract}
In this paper, the processes expressed with verbs of movement in Chanka Quechua, from the Ayacucho area, are briefly presented. Since Quechua is an agglutinative language, the aim of this paper is to analyze the function of suffixes from the semantic point of view. For this purpose, our theoretical framework is based on the contribution of cognitive semantics and on the relationship between semantics and grammar proposed by Moreno Cabrera (2003). The results of this work aim to classify and determine how the suffixes -man, -manta, -kama and -ta act in sentence constructions of movement.
\end{abstract}

Keywords: cognitive semantics, formal semantics, process, verbs of movement, verbs of displacement.

\section{Resumo}

Neste documento, são brevemente apresentados os processos expressos com verbos de movimento em Chanka Quechua, da área de Ayacucho. Sendo o quíchua uma linguagem aglutinativa, o objectivo deste trabalho é analisar a função dos sufixos do ponto de vista semântico. Para este efeito, o nosso quadro teórico baseia-se na contribuição da semântica cognitiva e na relação 
El estudio de los procesos con verbos de movimiento en el quechua chanka Mery Eli Ascencio Huaccho y Raquel Herlinda Román Barrera

entre semântica e gramática proposta por Moreno Cabrera (2003). Os resultados deste trabalho têm como objectivo classificar e determinar como os sufixos -man, -manta, -kama e -ta agem em construções de frases de movimento.

Palavras-chave: semântica cognitiva, semântica formal, processo, verbos de movimento, verbos de deslocamento.

\section{Introducción}

Los verbos de movimiento establecen relación con una entidad, pues el movimiento dependerá de alguien o algo. La locución verbos de movimiento es controversial, debido a que se distingue entre los verbos de desplazamientos y los verbos de movimiento. Los verbos de desplazamiento son considerados como acciones extrínsecas al sujeto, mientras los verbos de movimiento son intrínsecos al sujeto. Las investigaciones de este tipo de verbos se han llevado a cabo en lenguas como el inglés, el español, el alemán, etc. En esta oportunidad, se intenta escarbar a estos verbos en la lengua quechua.

La lengua quechua se caracteriza por ser aglutinante, es decir, construye palabras y oraciones al añadir sufijos. La variedad quechua a analizar corresponde al Quechua II (denominado por Alfredo Torero, 1964) y perteneciente al Quechua C (clasificado así por Gary Parker, 1963). La pregunta principal de este trabajo es ¿cuáles son los sufijos que acompañan a las construcciones oracionales de movimiento y cuál es su función? Asimismo, el objetivo es determinar las clasificaciones de los verbos de movimiento o desplazamiento de acuerdo con las propuestas desarrolladas por autores como Talmy, Jackendoff, Lamiroy, Cifuentes y Llopis, y Morimoto. También, se describirá la intervención de los sufijos en construcciones oracionales de movimiento y se determinarán los rasgos de cada verbo.

En cuanto al marco teórico, está compuesto por las posturas desarrolladas por los autores mencionados. A partir de ello, se clasificará el corpus compuesto por dieciocho oraciones quechua con su respectiva traducción. También, se presentan los resultados, la discusión del análisis y las conclusiones.

Finalmente, nuestro estudio se limita a analizar oraciones simples del quechua chanka que incluyen verbos de movimiento. Debido a la situación causada por la Covid-19 solo se contó con la participación de un colaborador, el docente de la UNMSM-FLCH Walter Chalco. 
El estudio de los procesos con verbos de movimiento en el quechua chanka Mery Eli Ascencio Huaccho y Raquel Herlinda Román Barrera

\section{Marco conceptual y teórico}

\subsection{Semántica y gramática}

La semántica formal considera necesario integrar el aspecto semántico en la gramática, pues no es correcto pensar en la sintaxis de manera independiente sin agregar las especificaciones del léxico. Uno de los fenómenos importantes de la sintaxis es «la organización de las frases en función de los verbos [...] [es decir] tomar en cuenta la diferencia que existe entre verbos transitivos, intransitivos, o verbos con preposición» (Cárdenas,2010).

Según la determinación del verbo, se conocerá si la construcción oracional expresa un proceso de desplazamiento o de mutación. En síntesis, Moreno Cabrera (2003) propone la siguiente estructura:

a. $\mathrm{P}[\Sigma(\Upsilon, \Phi), \Sigma(\Upsilon, \Theta)]$

b. $\Sigma(\Upsilon, \Phi) \rightarrow \sum(\Upsilon, \Theta)$

Esta formalización corresponde al proceso de desplazamiento o de mutación, donde $(\Sigma)$ es el estado de la entidad $(\Upsilon)$ en un lugar inicial $(\Phi)$ que mediante un proceso $(\rightarrow)$ el estado será la entidad en un lugar final $(\Theta)$.

\subsection{Semántica formal}

La semántica formal intenta caracterizar el significado por medio de la aplicación de "condiciones de verdad» sea en modelos o mundos posibles, la cual requiere del uso de herramientas o técnicas lógico-matemáticas. Asimismo, la semántica formal considera el pensamiento como proposicional, es decir, funciona como una lengua (Pires, 2001). Veamos el siguiente ejemplo de Pires (2001).

(1) a. Todo ser humano é mortal.

b. João é homem.

c. João é mortal.

(2) a. Todos os cachorros são chatos.

b. Munique é uma cachorro.

c. Munique é chata. 
El estudio de los procesos con verbos de movimiento en el quechua chanka Mery Eli Ascencio Huaccho y Raquel Herlinda Román Barrera

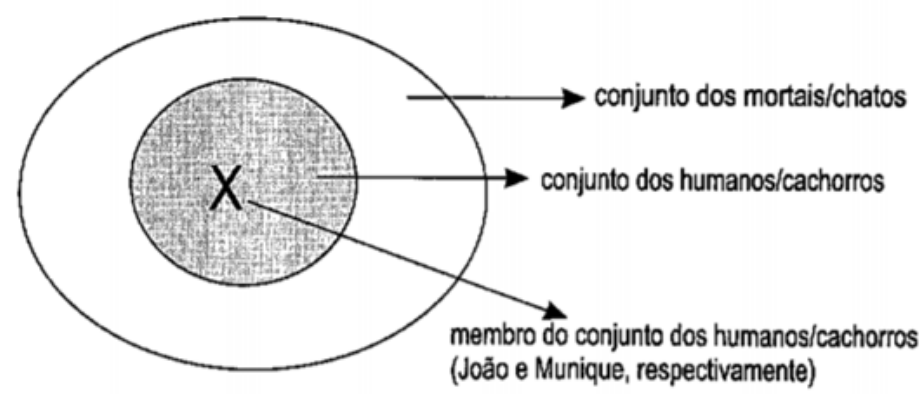

(Pires, 2001, p.29).

Para establecer la relación que mantienen estas proposiciones, Pires utiliza la teoría de conjuntos, en la cual X es una entidad que está dentro de un conjunto $\mathrm{H}$ (círculo gris) y a su vez, este conjunto está dentro de otro conjunto $\mathrm{M}$ (círculo blanco). Esta relación se expresa $\mathrm{X} \in \mathrm{H}$ e $\mathrm{H} \subset \mathrm{M}$. Notamos que João pertenece al conjunto de humanos que a su vez está contenido en conjunto de mortales, mientras Munique pertenece al conjunto de cachorros y al conjunto de aburridos (chatos). Esta descripción formal permite conocer la relación entre proposiciones y las condiciones de verdad.

Por otra parte, existe una relación entre la entidad y sus cambios de estados, la cual se denomina proceso. Para Moreno (2003), «los procesos se definen como relaciones de transición entre estados. Hay un proceso cuando una entidad, de estar en un estado pasa estar en otro estado [...] dichos estados comparten la misma entidad» (p. 103). Existen dos tipos de estados: localizaciones y atribuciones. Sin embargo, para este trabajo es imprescindible conocer los estados locativos también conocidos como desplazamientos. Los verbos de movimiento o desplazamiento poseen una trayectoria como parte inherente de su significado. Ej.:

(3) a. Pedro camina desde Lince hasta San Borja. 
El estudio de los procesos con verbos de movimiento en el quechua chanka Mery Eli Ascencio Huaccho y Raquel Herlinda Román Barrera

Estructura semántica

b. P [ $\Sigma^{\text {ad }}$ (Pedro, Lince), $\Sigma^{\text {ad }}$ (Pedro, San Borja) $]$

c. $\sum^{\mathrm{ad}}$ (Pedro, Lince) $\rightarrow \sum^{\mathrm{ad}}$ (Pedro, San Borja)

Estas estructuras semánticas expresan que una entidad (Pedro) se encuentra en un lugar determinado (Lince), por ello, se expresa como estado inesivo $\sum^{\mathrm{IN}} \mathrm{y}$ como consecuencia de la acción del verbo «caminar», la entidad se desplazará a un nuevo punto (San Borja). El recorrido de un estado inicial hacia un estado final es lo que se conoce como proceso. Además, entre el estado inicial y el estado final se encuentran estados intermedios que serían los lugares por los que se desplazó la entidad desde Lince hasta San Borja.

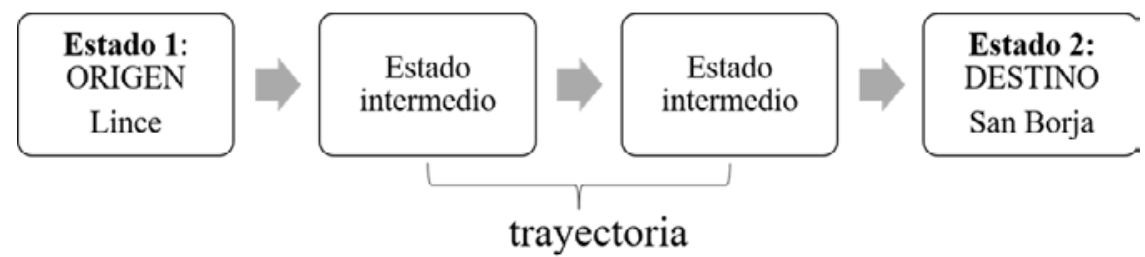

\subsection{Tipos de desplazamiento}

Existen cinco tipos de desplazamiento de manera general, aunque de acuerdo a la morfología de la lengua esto puede variar. Tal como sucede en el húngaro que presenta desplazamientos específicos pertenecientes a los cinco tipos de desplazamientos que se procederá a definir.

- Original inesivo

Este tipo de desplazamiento consiste en que la entidad presenta un punto de origen determinado. Por ejemplo, en la oración Marta baja del segundo piso de la casa indica que Marta tiene un estado de origen en el segundo piso de la casa. Semánticamente se representa así: P [ $\Sigma^{\text {sup }}$ (Marta, segundo piso), $\left.\Sigma^{\mathrm{x}}(\operatorname{Marta}, \wedge)\right]$.

- Original exesivo 
El estudio de los procesos con verbos de movimiento en el quechua chanka Mery Eli Ascencio Huaccho y Raquel Herlinda Román Barrera

El punto de origen del desplazamiento original exesivo está delimitado por la dirección elegida por la entidad con relación a un lugar, por ejemplo, en Marta ha venido de este lado de Ica, la entidad eligió un determinado lado del punto de origen para llegar a su destino. Semánticamente se representa así: $\mathrm{P}\left[\Sigma^{\mathrm{cit}}\left(\right.\right.$ Marta, Ica), $\left.\Sigma^{\mathrm{X}}(\operatorname{Marta}, \Lambda)\right]$.

- Destinativo inesivo

El desplazamiento inesivo presenta un punto de destino determinado. Por ejemplo, Marta ingresa en la oficina, se desconoce el punto de partida, pero se conoce que el destino de Marta culmina en la oficina. Semánticamente se representa así: $\mathrm{P}\left[\sum^{\mathrm{Xt}}(\operatorname{Marta}, \Lambda), \Sigma^{\mathrm{int}}(\right.$ Marta, oficina) $)$.

- Destinativo exesivo

Similar al desplazamiento original exesivo, la entidad elige una dirección por la cual recorren, en este caso está relacionado al punto final o destino. Por ejemplo, Marta ha ido a este lado de la casa. Semánticamente se representa así: $\mathrm{P}\left[\Sigma^{\text {cit }}\left(\right.\right.$ Marta, casa), $\Sigma^{\mathrm{X}}($ Marta, $\left.\Lambda)\right]$.

- Original-destinativo

El desplazamiento original-destinativo presenta un punto de partida y un punto final de la trayectoria. Ver la oración $\mathrm{N}^{\circ} 3$.

\subsection{Semántica cognitiva}

La semántica cognitiva tiene origen en la semántica generativa. Posteriormente, se autonomiza, pero tienen en común el «incorporar [...] el significado al análisis sintáctico» (Valenzuela, Ibarretxe y Hilfertly, 2012, p.35). La semántica cognitiva otorga una gran importancia al significado, por ello, no asume que el significado sea algo «externo» al mundo. Dada esta importancia, la sc está en la cabeza del conceptualizador, es decir, en la persona (Valenzuela, Ibarretxe y Hilfertly, 2012).

\subsection{Análisis de Talmy (1985 y 2001)}

Talmy establece que las lenguas se formalizan de manera distinta según los componentes de una escena de movimiento. El autor afirma que «los verbos de manera 
El estudio de los procesos con verbos de movimiento en el quechua chanka Mery Eli Ascencio Huaccho y Raquel Herlinda Román Barrera

de movimiento del español prácticamente no son compatibles con indicaciones de trayectoria (como flotar...)» (como se citó en Cuartero, 2006). Sin embargo, esta conclusión deja cierta incertidumbre, ya que a simple vista el verbo «flotar» presupone una trayectoria. Talmy desarrolla tres conceptos semánticos básicos de desplazamiento, a partir de ello determinará las funciones que cumplen los elementos sintácticos. En la Tabla 1 se sintetizan los conceptos básicos utilizados por Talmy.

Tabla 1. Componentes semánticos básicos de desplazamiento según Talmy

\begin{tabular}{l|l|l|}
\multicolumn{3}{c}{ Desplazamiento: La presencia del desplazamiento } \\
\hline Figura: Trayector & Trayectoria: Trayecto & Fondo: Locus \\
\hline $\begin{array}{l}\text { El objeto que se mueve } \\
\text { con respecto a otro } \\
\text { que sirve de punto de } \\
\text { referencia, que vendría a } \\
\text { ser el fondo. }\end{array}$ & $\begin{array}{l}\text { El curso seguido por la } \\
\text { figura con respecto al } \\
\text { punto de referencia. }\end{array}$ & $\begin{array}{l}\text { El objeto-punto de } \\
\text { referencia con respecto al } \\
\text { cual se mueve la figura. }\end{array}$ \\
\hline
\end{tabular}

Nota: Tomado de Pérez (2008, p.58).

En la oración:
(4) Marta
sube
$\mathrm{SN}$
$\mathrm{V}$
hacia la montaña.
(Figura)
(Trayectoria)
SN
(Fondo)

El verbo subir expresa el desplazamiento de los SN Marta y montaña que corresponde a la Figura y Fondo, respectivamente. La preposición hacia expresa una trayectoria determinada en relación con el Fondo o ubicación.

\subsection{Análisis de Aske (1989)}

La propuesta de Aske refuta lo planteado por Talmy, pues, si se asume de manera estricta el análisis de Talmy, muchos verbos de manera de desplazamiento no tendrían una explicación de su trayectoria, como sucede con el verbo flotar. Por ello, Aske establece condiciones aspectuales en los verbos. Se distingue en dos modos de organización: los télicos y los atélicos. 
El estudio de los procesos con verbos de movimiento en el quechua chanka Mery Eli Ascencio Huaccho y Raquel Herlinda Román Barrera

Trayectorias télicas

- Trayectoria de destino a ([Objeto/ Ubicación])]

- Trayectoria de origen: [de([Objeto/ Ubicación] $)]$

Trayectorias atélicas

- Trayectoria de tránsito: [vía([Objeto/ Ubicación])]

- Trayectoria de extensión: [por ([Objeto/ Ubicación])]

- Trayectoria extensiva con límite final: [hasta([Objeto/ Ubicación])]

- Trayectoria extensiva con límite inicial: [desde([Objeto/ Ubicación])]

Como menciona Cuartero (2006) «de este análisis se desprende igualmente que existe una distribución neta de los dos tipos de verbos: los de manera de desplazamiento se caracterizan frente a los de desplazamiento por su incompatibilidad con los complementos que indican meta (destino) u origen (p.17)».

Este esquema resuelve el problema de los verbos de manera de movimiento en enunciados como "*anduvo a casa o *nadó de la orilla (Cuartero,2006, p.17)», ya que al presentar la preposición $a$ y de, estos enunciados son considerados de trayectoria télica. Sin embargo, estos mismos enunciados son ambiguos al presentar la preposición hasta como en anduvo hasta su casa o nadó hasta la orilla, que por la presencia de la preposición hasta, los enunciados mencionados son considerados de trayectoria atélica.

\subsection{Análisis de Jackendoff (1990)}

Jackendoff clasifica dos tipos de verbos: de desplazamiento (VVDD) y los de manera de movimiento (VMMs) en su Estructura Léxico-Conceptual (ELC). Los vvDD están conformados por la función $I R$, el Objeto y la Trayectoria, mientras los VMMs se basan en la función MOVERSE e introduce al Objeto.

Verbos de desplazamiento (VD): $\left[_{\text {EVento }}\right.$ IR $\left.\left(\left[_{\text {OBjeto }}\right],\left[_{\text {TrAyectoria }}\right]\right)\right]$

Verbos de manera de moverse (VMM): $\left[_{\text {eVento }} \operatorname{MOVERSE}\left(\left[_{\text {овjeto }}\right]\right)\right]$

Únicamente, los verbos de desplazamiento son considerados aquellos que tienen trayectoria. Cabe mencionar, que Jackendoff alude a los verbos de trayectoria como Path y distingue verbos implícitos y no explícitos, es decir, verbos con o sin implicaciones del movimiento interno con respecto a su ubicación. Jackendoff define Path como: 
El estudio de los procesos con verbos de movimiento en el quechua chanka Mery Eli Ascencio Huaccho y Raquel Herlinda Román Barrera

Similarly elaborates a path, or trajectory, as one of five functions that map a reference thing or place into a related trajectory. An example of a path with a reference thing is to the house, an example of a path with a reference place is form under the table, where the trajectory begins at the place «under the table» (p.44).

La propuesta de Jackendoff se atañe parcialmente en la propuesta de Talmy, la cual no establece un criterio sobre la ubicación de verbos como «run», «walk» o «swin». Incluso, aplica la regla Adjunct-Go que permite describir un desplazamiento a partir de un verbo de modo de movimiento.

\subsection{Análisis de Lamiroy (1991)}

Lamiroy distingue verbos de dirección y verbos de desplazamiento. Con respecto a los primeros «indican un desplazamiento orientado, polarizado por un punto determinado que [...] es constitutivo del sentido del verbo» frente a los de desplazamiento, el cual «no se hace por referencia a un punto determinado por la posición del hablante y por la geometría del espacio» (Cuartero,2006).

\subsection{Análisis de Cifuentes y Llapis (2000)}

Según Cifuentes y Llapis, los verbos de desplazamiento son inherentemente télicos. Estos verbos siempre implican una localización y llevan cualquier preposición direccional final (Voy a/ hacia/ hasta casa).

En cambio, los verbos de modo de desplazamiento son inherentemente atélicos y pueden predicarse sin el componente incidencia espacial Al tener mayor compatibilidad con los complementos que indiquen camino, presentan usos prepositivos que manifiestan dicha relación. Finalmente, solo aceptan las de contenido más concreto, hacia y hasta (Cuartero, 2006).

\subsection{Análisis de Morimoto (2001)}

El análisis de Morimoto cuestiona la regla Adjunct-GO aplicada en el español. No obstante, considera una reinterpretación de la propuesta de Jackendoff. Morimoto está de acuerdo con Jackendoff en la distinción entre los vvdD y los vmms. Aunque, la autora clasifica los vmms en dos grupos: los vmMs-E (movimiento con referencia externa) del tipo caminar y los vMms-I (movimiento interno) o del tipo tambalearse (Luo, 2016). El siguiente cuadro indica los rasgos de los tipos de verbos: 
El estudio de los procesos con verbos de movimiento en el quechua chanka Mery Eli Ascencio Huaccho y Raquel Herlinda Román Barrera

Tabla 2. Clasificación de verbos de movimiento según Morimoto

\begin{tabular}{l|l|l|l} 
& $\begin{array}{l}\text { Presencia de } \\
\text { desplazamiento }\end{array}$ & Trayectoria & $\begin{array}{l}\text { Manera de } \\
\text { moverse }\end{array}$ \\
\hline I. vVDD & Sí & Determinada & No \\
\hline IIa. VMMS-E & Sí & Indeterminada & Sí \\
\hline IIb. VMMS-I & No & Ausente & sí \\
\hline
\end{tabular}

Nota: Morimoto, 2001a (citado en Luo, 2016, p. 37)

Para Morimoto, los VVDD expresan desplazamiento con una determinada dirección y no contiene información sobre la manera de moverse, mientras que los vмMs solo indican un desplazamiento sin una trayectoria concretada.

\subsection{Análisis de Cuartero (2006)}

La clasificación de Cuartero es la síntesis y la superación de las demás propuestas mencionadas. Cuartero toma en cuenta la diferencia entre verbos de cambios de posición frente a los verbos de desplazamiento, entre verbos de movimiento del sujeto y de objeto y los verbos con objeto de lugar, es decir con complemento directo no afectado.

Tabla 3. Clases de verbos de movimiento del español

\begin{tabular}{l|l|l|l|l}
\hline \multicolumn{5}{|c}{ Movimiento } \\
\hline Cambio de posición & Desplazamiento \\
\hline Del suJ & Del CD & Del CD & Del sUJ & \multicolumn{2}{l}{} \\
\hline Intransitivos & $\begin{array}{l}\text { Transitivos co } \\
\text { objeto afectado }\end{array}$ & $\begin{array}{l}\text { Transitivos con } \\
\text { objeto afectado }\end{array}$ & intransitivos & $\begin{array}{l}\text { Transitivos } \\
\text { con objeto no } \\
\text { afectado }\end{array}$ \\
\hline $\begin{array}{l}\text { Levantarse } \\
\text { girarse }\end{array}$ & $\begin{array}{l}\text { Girar algo, } \\
\text { Levantar algo }\end{array}$ & $\begin{array}{l}\text { Llevar algo, } \\
\text { Transportar } \\
\text { algo }\end{array}$ & Salir, correr & $\begin{array}{l}\text { Recorrer algo, } \\
\text { rodear algo }\end{array}$ \\
\hline
\end{tabular}

Nota: Adaptado de Cuartero (2003, p. 151).

De los análisis y propuestas mencionadas, los verbos de desplazamiento serán clasificados según las perspectivas de cada autor mencionado.

164 Lengua y Sociedad. Revista de Lingüística Teórica y Aplicada 
El estudio de los procesos con verbos de movimiento en el quechua chanka Mery Eli Ascencio Huaccho y Raquel Herlinda Román Barrera

\section{Metodología}

El presente trabajo es de tipo descriptivo.

\subsection{Instrumento}

Los instrumentos utilizados fueron diccionarios de la gramática quechua, fichas bibliográficas y laptop para registrar la información.

\subsection{Elaboración del corpus}

Para la elaboración del corpus tomamos como referencia una lista de verbos de movimiento en español y de aquella extrajimos algunos verbos, con los cuales elaboramos oraciones simples siguiendo la gramática del quechua chanka. Luego de eso, corroboramos las construcciones oracionales con el apoyo del docente Walter Chalco, quien debido a la situación actual de la pandemia resultó ser nuestro único colaborador. Al final, el corpus quedó conformado por dieciocho oraciones con su respectiva segmentación morfológica.

\section{Tabla 4. Construcciones oracionales en quechua}

\begin{tabular}{|l|l|l|}
\hline N. & Oraciones en quechua & Traducción \\
\hline 1. & Juan raymi-man chaya-rqa-n. & «Juan llegó a la fiesta». \\
\hline 2. & $\begin{array}{l}\text { Nuqa chay chiru-manta lasta-pa } \\
\text { chaya-rqa-ni. }\end{array}$ & $\begin{array}{l}\text { «Yo he llegado de este lado de la } \\
\text { nevada». }\end{array}$ \\
\hline 3. & $\begin{array}{l}\text { Runa pata pata-ta qata-na-man } \\
\text { siqa-rqa-n. }\end{array}$ & $\begin{array}{l}\text { «El hombre subió la escalera a la } \\
\text { azotea». }\end{array}$ \\
\hline 4. & Yanu-na-man ri-chka-ni. & «Estoy yendo hacia la cocina». \\
\hline 5. & Maria Qosqo-manta Lima-man ri-n. & «María va de Cusco a Lima». \\
\hline 6. & Yachachiq salun-man yayku-rqa-n. & «El profesor entró en el salón». \\
\hline 7. & $\begin{array}{l}\text { Waway (churiy) Piura-man kunan } \\
\text { pawa-n. }\end{array}$ & «Mi hijo vuela ahora hacia Piura». \\
\hline 8. & Rosa wasi-manta lluqsi-n. & «Rosa sale de la casa». \\
\hline 9. & Qamkuna urqu-n-ta uraykamu-nkichik. & «Ustedes bajan por el cerro». \\
\hline 10. & $\begin{array}{l}\text { Albirtu mayu-manta chakra-man } \\
\text { chimpa-rqa-n. }\end{array}$ & «Alberto cruzó del río a la chacra». \\
\hline 11. & $\begin{array}{l}\text { Dansaqkuna kaylla-manta Ancash-pa } \\
\text { hamu-rqa-nku. }\end{array}$ & $\begin{array}{l}\text { «os danzantes han venido desde } \\
\text { cerca de Áncash». }\end{array}$ \\
\hline
\end{tabular}


El estudio de los procesos con verbos de movimiento en el quechua chanka Mery Eli Ascencio Huaccho y Raquel Herlinda Román Barrera

\begin{tabular}{|l|l|l|}
\hline N. & Oraciones en quechua & Traducción \\
\hline 12. & $\begin{array}{l}\text { Tayta mama-y Ayacucho-pa kaylla- } \\
\text { n-man puri-rqa-nku. }\end{array}$ & $\begin{array}{l}\text { «Mis padres han caminado cerca de } \\
\text { Ayacucho». }\end{array}$ \\
\hline 13. & Ana chaka kaylla-n-ta chimpa-n. & «Ana atraviesa cerca del puente». \\
\hline 14. & Sipas llaqta-manta hamun. & «La señorita viene del pueblo». \\
\hline 15. & Juan yachay wasi-man-(mi) kallpa-n. & «Juan corre hacia la escuela». \\
\hline 16. & Paykuna hampara-man siqa-ku-nku. & «Ellos se suben a la mesa». \\
\hline 17. & Qam wasiy-kama purinki. & «Tú caminas hasta mi casa». \\
\hline 18. & Nuqa Punokama richkani. & «Estoy yendo hasta Puno». \\
\hline
\end{tabular}

\section{Análisis}

(1) Juan raymiman chayarqan.

Juan raymi- man chaya- rqa $-n$.

N. N. 'fiesta' ILAT. 'a, hacia' V.'llegar allá' suf.PAs.exP suf. V.3P.S

Significado literal: Juan a la fiesta llegó.

=Juan llegó a la fiesta.

Formalización:

Destinativo inesivo

- $\quad \sum^{\mathrm{x}}($ Juan, $\Lambda) \rightarrow \sum^{\mathrm{in}}$ (Juan, raymi)

- Juan $\left(\Lambda^{\mathrm{x}}\right.$, raymi $\left.^{\mathrm{in}}\right)$

(2) Nuqa chay chirumanta lastapa chayarqani.

Nuqa chay chiru -manta lasta -pa

PRON. 1P. DEM. ADV. 'lado' ABL. N. GEN.

chaya rqa -ni.

V.venir' SUF.PAS.EXPER 1P.S

$=$ Yo he llegado de este lado de la nevada.

Formalización:

Original exesivo

- $\quad \sum^{\text {cit }}($ Ñuqa, lasta $) \rightarrow \sum^{x}(\tilde{N} u q a, \Lambda)$

- $\quad$ Nuqa (lasta ${ }^{\mathrm{cit}}, \Lambda^{\mathrm{x}}$ ) 
El estudio de los procesos con verbos de movimiento en el quechua chanka Mery Eli Ascencio Huaccho y Raquel Herlinda Román Barrera

(3) Runa pata patata qatanaman siqarqan.

Runa pata pata -ta qata -na - man

$\mathrm{N}$ 'hombre' N. 'escalera' Acus. V. 'cubrir, techar' NOM. ILAT 'a, hacia' Siqa-rqa

$-n$.

V. 'subir' SUF.PAS.EXP. SUF V.3P.S

Significado literal: 'El hombre a la escalera al techo subió.

$=$ El hombre subió la escalera a la azotea.

Formalización:

Destinativo inesivo

- $\quad \mathcal{N}\left(\right.$ Runa $\left[\sum^{\mathrm{x}}\right.$ (pata pata, $\left.\Lambda\right) \rightarrow \sum^{\mathrm{in}}$ (pata pata, qatana) $\left.]\right)$

(4) Yanunaman richkani

Yanu -na -man ri $\quad$-chka - ni.

V. 'cocinar' NOM. ILAT 'a, hacia' V.'ir' PROG SUf.1P.S

Significado literal: Hacia la cocina estoy yendo.

$=$ Estoy yendo hacia la cocina.

Formalización:

Destinativo inesivo

- $\quad \sum^{\mathrm{x}}(\tilde{N} u q a, \Lambda) \rightarrow \sum^{\text {in }}(\tilde{N} u q a, y a n u n a)$

- $\tilde{N} u q a\left(\Lambda^{\mathrm{x}}\right.$, yanuna $\left.^{\mathrm{in}}\right)$

(5) Maria Qosqomanta Limaman rin.

Maria Qosqo -manta Lima -man ri $-n$.

N. N. ABL. N Ilat. 'a, hacia' V. 'ir' 3P.S

=María va de Cusco a Lima

Formalización:

Original - destinativo

- $\quad \sum^{\text {in }}$ (María, Qosqo) $\rightarrow \sum^{\text {in }}$ (María, Lima) 
El estudio de los procesos con verbos de movimiento en el quechua chanka Mery Eli Ascencio Huaccho y Raquel Herlinda Román Barrera

(6) Yachachiq salunman yaykurqan.

Yachachiq salun -man

N. 'profesor' N. 'salón' ILAT 'a, hacia'

yayku -rqa $-n$

V. 'entrar' SUF. PAS.EXP. SUF V.3P.S

Significado literal: El profesor hacia el salón entró.

$=$ El profesor entró en el salón.

Formalización:

Destinativo inesivo

- $\quad \sum^{\mathrm{x}}$ (Yachachiq, $\left.\Lambda\right) \rightarrow \sum^{\text {in }}$ (Yachachiq,salun)

- Yachachiq $\left(\Lambda^{\mathrm{x}}\right.$, salun $\left.{ }^{\text {in }}\right)$

(7) Waway (churiy) Piuraman kunan pawan.

Waway (churiy) Piura -man kunan pawa -n.

N. N. ILAT. 'a, hacia' ADV. V. 'volar' suf.3P.s

$=$ Mi hijo vuela ahora hacia Piura.

Formalización:

Destinativo exesivo

- $\quad \sum^{\mathrm{x}}$ (Churiy, $\left.\Lambda\right) \rightarrow \sum^{\mathrm{sub}}$ (Churiy ,Piura)

- Churiy $\left(\Lambda^{\mathrm{x}}, \mathrm{Piuraman}^{\mathrm{sub}}\right)$

(8) Rosa wasimanta lluqsin.

Rosa wasi -manta lluqsi -n.

N. N.'casa' ablat. 'de, desde' V. 'salir' suf v.3P.s

Significado literal: Rosa desde la casa sale.

$=$ Rosa sale de la casa.

Formalización:

Original inesivo

- $\quad \sum^{\text {in }}($ Rosa,wasi $) \rightarrow \sum^{\mathrm{x}}($ Rosa,$\Lambda)$

- Rosa (wasi ${ }^{\mathrm{in},} \Lambda^{\mathrm{x}}$ ) 
El estudio de los procesos con verbos de movimiento en el quechua chanka Mery Eli Ascencio Huaccho y Raquel Herlinda Román Barrera

(9) Qamkuna urqunta uraykamunkichik

Qamkuna urqu -n $\quad$-ta uraykamu -nkichik.

PRON.2P.P N. 'cerro' 3P.POS ACUS. V. 'bajar por' SUF.2P.PL

$=$ Ustedes bajan por el cerro.

Formalización:

Destinativo inesivo

- $\quad \sum^{\mathrm{x}}($ Qamkuna, $\Lambda) \rightarrow \sum^{\mathrm{in}}$ (Qamkuna,urqu)

- Qamkuna $\left(\Lambda^{\mathrm{x}}, \mathrm{urqu}^{\mathrm{in}}\right)$

(10) Albirtu mayumanta chakraman chimparqan.

Albirtu mayu -manta chakra -man

N. N. 'río' ABL. 'de,desde' N. ILAT 'a, hacia'

chimpa -rqa $-\mathrm{n}$.

V.'cruzar' sUF..pas.exper sUf.3P.PL.

=Alberto cruzó del río a la chacra.

Formalización

Original - destinativo

- $\quad \sum^{\text {in }}$ (Albirtu, mayu) $\rightarrow \sum^{\text {in }}$ (Albirtu, chakra)

(11) Dansaqkuna kayllamanta Ancashpa hamurqanku.

Dansaquna kaylla -manta Ancash -pa

N. ADV. 'cerca' ABL. 'de,desde' N. GEN.

hamu rqa -nku.

V. 'venir' SUF.PAS.EXP. 3P.PL.

=Los danzantes han venido desde cerca de Áncash.

Formalización:

Original exesivo

- $\quad \sum^{\mathrm{ad}}$ (Dansaqkuna, Áncash) $\rightarrow \sum^{\mathrm{x}}$ (Dansaqkuna, $\left.\Lambda\right)$

- Dansaqkuna (Áncash ${ }^{\mathrm{ad}}, \Lambda^{\mathrm{x}}$ ) 
El estudio de los procesos con verbos de movimiento en el quechua chanka Mery Eli Ascencio Huaccho y Raquel Herlinda Román Barrera

(12) Tayta mamay Ayacuchopa kayllanman purirqanku.

Taytamama -y Ayacucho -pa kaylla-n-man

N. SUf.Pos.1P N. $\quad$ GEN. ADV. 'cerca'

Puri -rqa -nku.

V. 'caminar' SUF.PAS.EXP. SUF.3P.PL

$=$ Mis padres han caminado cerca de Ayacucho.

Formalización:

Destinativo exesivo

- $\quad \sum^{\mathrm{ad}}$ (Tayta mamay,Ayacucho) $\rightarrow \sum^{\mathrm{x}}$ (Tayta mamay, $\Lambda$ )

- Tayta mamay (Ayacucho ${ }^{\mathrm{ad}}, \Lambda^{\mathrm{x}}$ )

(13) Ana chaka kayllanta chimpan.

Ana chaka kaylla $-n$-ta chimpa $-n$.

N. N. 'puente' ADV. 'cerca' 'su' Acus. V. 'atravesar' 3P.S

$=$ Ana atraviesa cerca del puente.

Formalización:

Destinativo exesivo

- $\quad \sum^{\mathrm{x}}($ Ana, $\Lambda) \rightarrow \sum^{\mathrm{ad}}$ (Ana, chaka)

- Ana $\left(\Lambda^{\mathrm{x}}\right.$, chaka $\left.^{\mathrm{ad}}\right)$

(14) Sipas llaqtamanta hamun

Sipas llaqta -manta hamu -n.

N. 'señorita' N. 'pueblo' ABL. 'de, desde' V. 'venir' -suf V.3P.s

Significado literal: La señorita desde el pueblo viene.

$=$ La señorita viene del pueblo.

Formalización:

Original inesivo

- $\quad \sum^{\text {in }}($ Sipas,llaqta $) \rightarrow \sum^{\mathrm{x}}\left(\right.$ Sipas, $\left.\Lambda^{\mathrm{x}}\right)$

- Sipas (llaqta $\left.{ }^{\text {in }}, \Lambda^{\mathrm{x}}\right)$ 
El estudio de los procesos con verbos de movimiento en el quechua chanka Mery Eli Ascencio Huaccho y Raquel Herlinda Román Barrera

(15) Juan yachay wasimanmi kallpan.

Juan yachay wasi -man $-(\mathrm{mi})$ kallpa $-n$

N. V. 'estudiar' N. 'casa' ABL. VAL. V.'correr' 3P.s.

$=$ Juan corre hacia la escuela.

Formalización:

Destinativo inesivo

- $\quad \sum^{\mathrm{x}}$ (Juan, $\left.\Lambda\right) \rightarrow \sum^{\text {in }}$ (Juan ,yachay wasi)

- Juan $\left(\Lambda^{\mathrm{x}}\right.$,yachay wasi $\left.{ }^{\mathrm{in}}\right)$

(16) Paykuna hamparaman siqakunku.

Paykuna hampara -man siqa -ku $-n k u$.

PRON.3P.PL N. 'mesa' -ILAT 'a, hacia' V. 'subir' REFL. SUF V.3P.PL

Significado literal: Ellos hacia la mesa se suben.

$=$ Ellos se suben a la mesa.

Formalización:

Destinativo inesivo

- $\quad \sum^{\mathrm{x}}$ (Paykuna, $\left.\Lambda\right) \rightarrow \sum^{\mathrm{in}}$ (Paykuna , hampara)

- Paykuna $\left(\Lambda^{\mathrm{x}}\right.$,hampara $\left.{ }^{\mathrm{in}}\right)$

(17) Qam wasiykama purinki.

Qam wasi -y $\quad$-kama puri $\quad$-nki.

PRON 1P. N.'casa' POS.1P. LIM. V.'caminar' 2P.S.

= Tú caminas hasta mi casa.

Formalización:

Destinativo inesivo

- $\sum^{\mathrm{x}}(\mathrm{Q} a \mathrm{~m}, \Lambda) \rightarrow \sum^{\text {in }}(\mathrm{Q} a \mathrm{~m}$, wasi)

- $\operatorname{Qam}\left(\Lambda^{\mathrm{x}}\right.$, wasi $)$ 
El estudio de los procesos con verbos de movimiento en el quechua chanka Mery Eli Ascencio Huaccho y Raquel Herlinda Román Barrera

(18) Ñuqa Punokama richkani.

Nuqa Puno -kama ri -chka -ni.

PRON.1P. N. LIM. V.'ir’ SUF.PROG. 1.P.s.

= Estoy yendo hasta Puno.

Formalización:

Destinativo inesivo

- $\quad \sum^{\mathrm{x}}(\tilde{\mathrm{N} u q a}, \Lambda) \rightarrow \sum^{\text {in }}(\tilde{N} u q a, P u n o)$

- $\tilde{N} u q a\left(\Lambda^{\mathrm{x}}, \mathrm{Puno}^{\mathrm{in}}\right)$

De acuerdo con las dieciocho oraciones propuestas, se agruparán los rasgos hallados según algunos verbos de movimiento en distintas oraciones.

c. Siqay: En la oración N. ${ }^{\circ}$ 16, el sufijo - man indica la direccionalidad del trayector paykuna, además modificando el Fondo, de acuerdo a los términos empleados por Talmy. En cambio, en la oración N. ${ }^{\circ}$ 3, nos indica una acción del tipo agente-paciente, además el morfema -man indica una heterolocomoción destinativa.

d. Uray: En el caso de la oración N. ${ }^{\circ} 9$ Qamkuna urqu-nta uraykamu-nkichik 'Ustedes bajan por el cerro', se observa que el verbo «uray» presenta una trayectoria de un punto $\mathrm{X}$ a un punto Y. En esta oración no está explícito los puntos de origen y destino, pero si la vía. Con respecto al acusativo - nta, que en esta oración se traduce como la preposición 'por', cumple una función de extensión de trayectoria similar al español 'caminar por el parque'.

e. Hamuy: En las oraciones N. ${ }^{\circ} 11$ y 14 presentan el sufijo direccional -manta y notamos que presenta el sema de [ \pm lejano]. En ambas oraciones, el verbo hamuy alcanza un destino, es decir, el hablante señala su ubicación y la llegada al lugar, aunque en estas oraciones no menciona el punto de origen.

f. Llusiy: En la oración N. ${ }^{\circ}$ 8, el desplazamiento marcado por el sufijo -manta modifica al fondo wasi debido a la ausencia de la trayectoria lluqsin. Morimoto define al verbo salir como aquel que indica

172 Lengua y Sociedad. Revista de Lingüistica Teórica y Aplicada 
El estudio de los procesos con verbos de movimiento en el quechua chanka Mery Eli Ascencio Huaccho y Raquel Herlinda Román Barrera

un desplazamiento que implica la superación de un límite (citado en Pérez,2008). El sujeto de esta oración Rosa se desplaza en el interior del objeto wasi.

g. Chimpay: En la oración N. ${ }^{\circ}$ 10, el sufijo -man marcaría el tránsito. Además, el verbo chimpay señala el desplazamiento del objeto 1 mayu al objeto 2 chakra (meta).

(10) Albirtu mayumanta [Trayectoria] chakraman chimparqan.

$\begin{array}{llll}\text { (figura) } & \text { (ox1) } & \text { (ox2) } & \text { (evento) } \\ \text { Alberto } & \text { río } & \text { chacra } & \text { cruzar }\end{array}$

\subsection{Resultados y discusión}

A partir de las oraciones revisadas con verbos de desplazamiento pertenecientes al quechua chanka, se observa que los marcadores de esta lengua son netamente morfológicos, además se caracterizan por su semanticidad en cuanto a la trayectoria, lo cual está presente de manera subyacente en el hablante. De acuerdo con la formalización elaborada, se agrupó en la Tabla 5 las oraciones según el tipo de desplazamiento.

Tabla 5. Clasificación según el tipo de desplazamiento

\begin{tabular}{l|l}
\hline Original inesivo & $\begin{array}{l}\text { Rosa wasimanta lluqsin. } \\
\text { Sipas llaqtamanta hamun. }\end{array}$ \\
\hline Original exesivo & $\begin{array}{l}\text { Nuqa chay chirumanta lastapa chayarqani. } \\
\text { Dansaqkuna kayllamanta Ancashpa hamurqanku. }\end{array}$ \\
\hline Destinativo inesivo & $\begin{array}{l}\text { Juan raymiman chayarqan. } \\
\text { Runa pata patata qatanaman siqarqan. } \\
\text { Yanunaman richkani. } \\
\text { Yachachiq salunman yaykurqan. } \\
\text { Qamkuna urqunta uraykamunkichik. } \\
\text { Juan yachay wasimanmi kallpan. } \\
\text { Paykuna hamparaman siqakunku. }\end{array}$ \\
\hline Destinativo exesivo & $\begin{array}{l}\text { Waway (churiy) Piuraman kunan pawan. } \\
\text { Tayta mamay Ayacuchopa kayllanman purirqanku. } \\
\text { Ana chaka kayllanta chimpan. }\end{array}$ \\
\hline Original-destinativo & $\begin{array}{l}\text { Maria Qosqomanta Limaman rin. } \\
\text { Albirtu mayumanta chakraman chimparqan. }\end{array}$ \\
\hline
\end{tabular}


El estudio de los procesos con verbos de movimiento en el quechua chanka Mery Eli Ascencio Huaccho y Raquel Herlinda Román Barrera

Por otro lado, este análisis contrastó el corpus con las propuestas de los autores mencionados. Uno de ellos es Aske quien propone la clasificación de trayectorias télicas y atélicas. A continuación, en la Tabla 6 clasificamos algunas oraciones.

Tabla 6. Clasificación del corpus según la propuesta de Aske

\begin{tabular}{|c|c|c|}
\hline \multirow[t]{2}{*}{ 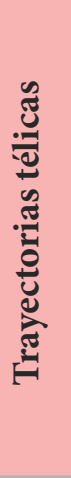 } & $\begin{array}{l}\text { Trayectoria de destino: } \\
\text { [a ([Objeto/ Ubicación] }])]\end{array}$ & $\begin{array}{l}\text { 1. Juan raymiman chayarqan. } \\
\text { 4. Yanunaman richkani. } \\
\text { 6. Yachachiq salunman yaykurqan } \\
\text { 7. Waway (churiy) Piuraman kunan } \\
\text { pawan. } \\
\text { 15. Juan yachay wasiman(mi) kallpan. } \\
\text { 16. Paykuna hamparaman siqakunku. }\end{array}$ \\
\hline & $\begin{array}{l}\text { Trayectoria de origen: } \\
\text { [de ([Objeto/ Ubicación])] }\end{array}$ & $\begin{array}{l}\text { 8. Rosa wasimanta lluqsin. } \\
\text { 14. Sipas llaqtamanta hamun. }\end{array}$ \\
\hline \multirow{4}{*}{ 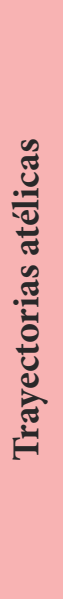 } & $\begin{array}{l}\text { Trayectoria de tránsito: } \\
\text { [vía ([Objeto/ Ubicación])] }\end{array}$ & $\begin{array}{l}\text { 5. Maria Qosqomanta Limaman rin. } \\
\text { 10. Albirtu mayumanta chakraman } \\
\text { chimparqan. }\end{array}$ \\
\hline & $\begin{array}{l}\text { Trayectoria de extensión: } \\
\text { [por ([Objeto/ Ubicación])] }\end{array}$ & 9. Qamkuna urqunta uraykamunkichik. \\
\hline & $\begin{array}{l}\text { Trayectoria extensiva con } \\
\text { límite final: } \\
\text { [hasta ([Objeto/ Ubicación])] }\end{array}$ & $\begin{array}{l}\text { 17. Qam wasiykama purinki. } \\
\text { 18. Nuqa Punokama richkani. }\end{array}$ \\
\hline & $\begin{array}{l}\text { Trayectoria extensiva con } \\
\text { límite inicial: } \\
\text { [desde ([Objeto/ Ubicación])] }\end{array}$ & $\begin{array}{l}\text { 2. Nuqa chay chirumanta lastapa } \\
\text { chayarqani. } \\
\text { 12. Dansaqkuna kayllamanta Ancashpa } \\
\text { hamurqanku. }\end{array}$ \\
\hline
\end{tabular}

La clasificación de Aske considera las preposiciones como aquellos que señalan si la construcción oracional es télica o atélica, lo cual objetamos. Una de las razones es porque la oración con sufijo -kama es clasificada como una trayectoria extensiva con límite final del tipo de trayectoria atélica. Se sabe que es el morfema -kama señala un límite, lo que significa la existencia de un punto final « $\mathrm{P}_{1} \rightarrow \mathrm{P}_{2} »$. Objetivamente, este sufijo indica un límite espacial-temporal y un determinado fin, en consecuencia, no debería pertenecer a la trayectoria atélica. De esta manera, estamos a favor de la discrepancia de Cuartero, quien indica lo siguiente: 
El estudio de los procesos con verbos de movimiento en el quechua chanka Mery Eli Ascencio Huaccho y Raquel Herlinda Román Barrera

Un aspecto del análisis que, sin embargo, no parece muy convincente es atribuir carácter atélico al menos a las indicaciones con hasta, ya que esta postura obliga al autor a aceptar que el enunciado «Juan caminó hasta la cima ( ${ }^{*}$ en dos horas)» ha de ser forzosamente atélico (cuando no lo es: ${ }^{\star} J u a n$ caminó hasta la cima durante dos horas), y a continuación a dar explicaciones un tanto confusas: «hasta, 'up to', 'until', for instance, contrary to what one might have thought, is not telic and doesn't predicate a final location of the Figure, that is, the final location is not asserted [cursiva mía], though it certanly may be implied» (2006,p.18).

Aunque, se debe tener en cuenta que Aske concibe el término «telicidad» como una "propiedad aspectual de los eventos que [implican] un determinado estado resultativo» (Morimoto, citado en Luo, 2006, p. 35). Es decir, «el concepto 'telicidad' se distingue claramente del de 'delimitación', porque una Trayectoria espacialmente delimitada no es necesariamente télica» (Luo, 2006, p. 35).

Otra de las clasificaciones que se tomó en cuenta es la de Morimoto.

Tabla 7. Clasificación según Morimoto

\begin{tabular}{|c|c|c|c|}
\hline & Presencia de desplazamiento & Trayectoria & $\begin{array}{l}\text { Manera de } \\
\text { moverse }\end{array}$ \\
\hline I. VVDD & $\begin{array}{l}\text { 5. Maria Qosqomanta Limaman rin. } \\
\text { 10. Albirtu mayumanta chakraman } \\
\text { chimparqan. }\end{array}$ & Determinada & No \\
\hline IIa. VMMS-E & $\begin{array}{l}\text { 4. Yanunaman richkani. } \\
\text { 11. Dansaqkuna kayllamanta } \\
\text { Ancashpa hamurqanku. } \\
\text { 15. Sipas llaqtamanta hamun. } \\
\text { 17. Qam wasiy-kama pur } \\
\text { 18. Nuqa Punokama richkani }\end{array}$ & Indeterminada & Sí \\
\hline IIb. VMMS-I & No hay desplazamiento espacial & Ausente & sí \\
\hline
\end{tabular}

De acuerdo con la clasificación de Morimoto, nuestro corpus solo presenta verbos de clase VvDD determinada y de clase vMMs-E como verbos kallpay 'correr' y puriy 'caminar'. En el caso de los VMMs-I, se sugiere continuar con la investigación. 
El estudio de los procesos con verbos de movimiento en el quechua chanka Mery Eli Ascencio Huaccho y Raquel Herlinda Román Barrera

La Tabla 8 representa la clasificación de los verbos según Cuartero, sin embargo, discrepamos, pues todos los verbos del quechua chanka son regulares. Por esta razón, el desplazamiento del sujeto tendría que dividirse en «transitivos» $\mathrm{y}$ «transitivos con objeto no afectado».

Tabla 8. Clasificación de los verbos según Cuartero (2006)

\begin{tabular}{l|l|l|}
\hline \multicolumn{3}{|c|}{ Movimiento } \\
\hline \multicolumn{1}{|c|}{ Desplazamiento } \\
\hline \multicolumn{1}{|c|}{ Del CD } \\
$\begin{array}{l}\text { Transitivos con objeto } \\
\text { afectado }\end{array}$ & $\begin{array}{l}\text { Intransitivos } \\
\text { (Transitivos) }\end{array}$ & $\begin{array}{l}\text { Transitivos con objeto } \\
\text { no afectado }\end{array}$ \\
\hline Siqay & $\begin{array}{l}\text { Chayay, kallpay, riy, } \\
\text { hamuy, lluqsiy, puriy, } \\
\text { yaikuy }\end{array}$ & Riy, paway \\
\hline
\end{tabular}

En la Tabla 9 se resume los verbos utilizados en relación a los rasgos que presentan.

Tabla 9. Rasgos semánticos de los verbos de movimiento

\begin{tabular}{l|l}
\hline $\begin{array}{l}\text { Verbos } \\
\text { Chayay = llegar }\end{array}$ & Rasgos \\
\hline Siqay = subir & $\begin{array}{l}\text { [proceso] [dinamismo] [movimiento] } \\
\text { [verticalidad] [tránsito] [hacia arriba] }\end{array}$ \\
\hline Riy = ir & $\begin{array}{l}\text { [proceso] [dinamismo] [movimiento] } \\
\text { [horizontalidad] }\end{array}$ \\
\hline Yaykuy = entrar & $\begin{array}{l}\text { [proceso] [dinamismo] [movimiento] } \\
\text { [horizontalidad] [tránsito] [interno] }\end{array}$ \\
\hline Paway = volar & $\begin{array}{l}\text { [proceso] [dinamismo] [movimiento] } \\
\text { [tránsito] [aire] }\end{array}$ \\
\hline Llusiy = Salir & $\begin{array}{l}\text { [proceso] [dinamismo] [movimiento] } \\
\text { [horizontalidad] [tránsito] [hacia fuera] }\end{array}$ \\
\hline Uray = bajar & $\begin{array}{l}\text { [proceso] [dinamismo] [movimiento] } \\
\text { [verticalidad] [tránsito] [hacia abajo] }\end{array}$ \\
\hline
\end{tabular}


El estudio de los procesos con verbos de movimiento en el quechua chanka Mery Eli Ascencio Huaccho y Raquel Herlinda Román Barrera

\begin{tabular}{l|l}
$\begin{array}{l}\text { Verbos } \\
\text { Chimpay }= \\
\text { cruzar }\end{array}$ & $\begin{array}{l}\text { Rasgos } \\
\text { [proceso] [dinamismo] [movimiento] } \\
\text { [verticalidad] [tránsito] }\end{array}$ \\
\hline Hamuy = venir & $\begin{array}{l}\text { [proceso] [dinamismo] [movimiento] } \\
\text { [verticalidad] [tránsito] [persona] }\end{array}$ \\
\hline Puriy = caminar & $\begin{array}{l}\text { [proceso] [dinamismo] [movimiento] } \\
\text { [verticalidad] [tránsito] [hacia delante] }\end{array}$ \\
\hline $\begin{array}{l}\text { Chimpay }= \\
\text { atravesar }\end{array}$ & $\begin{array}{l}\text { [proceso] [dinamismo] [movimiento] } \\
\text { [verticalidad] [tránsito] }\end{array}$ \\
\hline Kallpay = correr & $\begin{array}{l}\text { [proceso] [dinamismo] [movimiento] } \\
\text { [horizontalidad] [tránsito] [hacia } \\
\text { adelante] [rapidez] }\end{array}$ \\
\hline
\end{tabular}

La Tabla 10 representa cuatro sufijos importantes para las oraciones que expresan desplazamiento.

En nuestro corpus, generalmente el sufijo -man expresa el tipo de desplazamiento destinativo inesivo.

En el caso del sufijo - manta expresa el tipo de desplazamiento original inesivo y original exesivo.

El sufijo -kama expresa el tipo de desplazamiento destinativo inesivo.

El sufijo - $t a$ expresa el tipo de desplazamiento destinativo inesivo y destinativo excesivo.

En oraciones de tipo de desplazamiento original-destinativo emplean los sufijos -man y -manta.

Tabla 10. Clasificación de los sufijos según su función (Zariquiey y Córdova, 2008)

\begin{tabular}{|c|c|c|c|}
\hline \multicolumn{4}{|l|}{ Sufijos } \\
\hline -Man & -Manta & -Kama & $-\mathrm{Ta}$ \\
\hline $\begin{array}{l}\text { Marca al objeto directo } \\
\text { de un verbo ditransitivo } \\
\text { o la dirección inexacta de } \\
\text { un verbo de movimiento. } \\
\text { Equivalente a las } \\
\text { preposiciones } a \text {, hacia } \\
\text { del castellano. }\end{array}$ & $\begin{array}{l}\text { Indica el origen o } \\
\text { punto de partida. } \\
\text { En castellano } \\
\text { equivale a las } \\
\text { preposiciones } \\
\text { desde, de, del. }\end{array}$ & $\begin{array}{l}\text { Expresa límite } \\
\text { en el tiempo y } \\
\text { en el espacio. }\end{array}$ & $\begin{array}{l}\text { Indica el objeto } \\
\text { directo de la } \\
\text { oración o el } \\
\text { punto de llegada } \\
\text { de un verbo de } \\
\text { movimiento, } \\
\text { como 'ir'. }\end{array}$ \\
\hline
\end{tabular}


El estudio de los procesos con verbos de movimiento en el quechua chanka Mery Eli Ascencio Huaccho y Raquel Herlinda Román Barrera

Finalmente, algunas características importantes de los verbos de movimiento es la diferencia entre verbos de dirección y verbos de desplazamientos propuesto por Lamiroy, también el carácter télico inherente de los verbos de desplazamiento y la implicancia de una localización, enfoque de Cifuentes y Llapis. Con respecto a la propuesta de Jackendoff sobre los verbos de desplazamiento o también sintetizado en su regla Adjunct-GO, al parecer los verbos contienen rasgos subyacentes del verbo 'ir', lo que correspondería a una función eventiva. Por otro lado, los estudios de Talmy deja cabos sueltos en relación a algunos verbos, consideramos que la presencia de un trayector, un trayecto y un locus corresponde a conceptos semánticos válidos para el corpus analizado.
Juan
yachay wasi
-man-(mi)
kallpa-n
(Figura)
(Fondo/Locus)
(Determinada)
(Trayectoria)

\section{Conclusiones}

A partir del corpus analizado se logra extrapolar la estrecha relación semántica-sintaxis en la lengua quechua chanka. Los sufijos marcadores de caso -manta, -kama, -man y -ta son añadidos a los verbos de movimiento y expresan el punto de partida y el punto de llegada, es decir, el tipo de desplazamiento sea original o destinativo.

Los sufijos -man, -manta y -kama son más económicos lingüísticamente, en el sentido de que los quechuahablantes prefieren los sufijos de lugar ya mencionados, que el sufijo - $t a$ de acuerdo a las oraciones revisadas.

Por otro lado, se recusa la propuesta de Aske en cuanto a su término «telicidad», por considerar a la preposición «hasta» parte de la trayectoria atélica, por ejemplo, la oración $\mathrm{N}^{\circ} 19$.

El análisis de Talmy se ajusta a nuestro corpus en cuanto a los términos figura, trayectoria y fondo, aunque debe verificarse la selectividad de Talmy con respecto a algunos verbos aplicados al corpus utilizado.

Los verbos propuestos para este estudio presentan los rasgos de [+proceso], [+dinamismo], [+movimiento], [+desplazamiento]. Los verbos como siqay 'subir' y uray 'bajar' presentan los rasgos de [+verticalidad] y verbos como yaykuy 'entrar' y lluqsy 'salir' presentan el rasgo de [+horizontalidad], es decir, el desplazamiento de un punto $\mathrm{A}$ a un punto $\mathrm{B}$, lo cual sería antagónico al rasgo [+verticalidad] de los verbos siqay y uray.

178 Lengua y Sociedad. Revista de Lingüística Teórica y Aplicada 
El estudio de los procesos con verbos de movimiento en el quechua chanka Mery Eli Ascencio Huaccho y Raquel Herlinda Román Barrera

Finalmente, para señalar en el quechua los tipos de localizaciones exesivas, se recurre al uso de adverbios como chiru 'lado' y kaylla 'cerca'. Estos adverbios son colocados a la izquierda de los sufijos que indican el punto de origen y el punto de llegada.

\section{Agradecimientos}

Los autores agradecen los alcances brindados por el lingüista Walter Chalco Arangoitia, profesor del curso de lengua originaria en la Universidad Nacional Mayor de San Marcos. También es oportuno señalar un agradecimiento a la profesora Verónica Lazo por sus observaciones y revisiones para la confección del presente manuscrito.

\section{Financiamiento}

La investigación se realizó sin financiamiento.

\section{Conflicto de intereses}

Los autores no presentan conflicto de interés. 
El estudio de los procesos con verbos de movimiento en el quechua chanka Mery Eli Ascencio Huaccho y Raquel Herlinda Román Barrera

\section{Referencias bibliográficas}

Chuquimamani, N. (2005). Yachakuqkunapa Simi Qullqa-Qusqu Qullaw Qhichwa Simipi. Ministerio de Educación

Cuartero, J. (2006). ¿Cuántas clases de verbos de desplazamiento se distinguen en español?

Ibarretxe, I., y Valenzuela, J. (2012). Lingüística Cognitiva: origen, principios y tendencias. Lingüística cognitiva, 13, 38.

Jackendoff, R. (1992). Estructuras semánticas (Vol. 18). Prensa del MIT.

Luo, Y. (2016). Verbos de desplazamiento en español y en chino: Un análisis subléxico de su significado y sus extensiones semánticas [Tesis doctoral].

Moreno, J. C. (2003). Semántica y gramática: sucesos, papeles semánticos y relaciones sintácticas. A. Machado.

Pérez Ríos, J. A. (2008). Análisis morfológico-semántico de los verbos de desplazamiento en taushiro, una lengua en extinción [Tesis de licenciatura, Universidad Nacional Mayor de San Marcos].

Pires, R. (2001). Semântica Formal. Uma Breve Introdução. Campinas: Mercado de Letras.

Valenzuela, J., Ibarretxe, I., y Hilferty, J. (2012). La semántica cognitiva. Lingüística cognitiva, dirs. I.

Zariquiey, R., y Córdova, G. (2008). Qayna, kunan, paqarin. Una introducción práctica al quechua chanca. Pontificia Universidad Católica del Perú. Estudios Generales Letras.

\section{Trayectoria académica de los autores}

Mery Eli Ascencio Huaccho es estudiante de la Escuela Profesional de Lingüística de la Universidad Nacional Mayor de San Marcos (UNMSM). Actualmente, cursa el octavo ciclo y sus intereses se orientan a la dialectología quechua, semántica, lexicología y lexicografía.

Raquel Herlinda Román Barrera estudia Lingüística en la Universidad Nacional Mayor de San Marcos (UNMSM). Actualmente, cursa el octavo ciclo y sus intereses se orientan al análisis del discurso, la semántica y la sociolingüística.

$180 \quad$ Lengua y Sociedad. Revista de Lingüística Teórica y Aplicada 